\title{
MECHANICAL DESIGN OF A FLEXIBLE BUNCH COMPRESSOR FOR APS LINAC*
}

\author{
E. Rotela ${ }^{\dagger}$, S. Sharma, A. Barcikowski, and J. Biggs \\ Advanced Photon Source, Argonne National Laboratory, Argonne, IL
}

\begin{abstract}
A bunch compressor has been installed in the APS linac to produce high peak currents for the FEL project and to explore the effects of coherent synchrotron radiation (CSR). The bunch compressor design is based on an asymmetric four-dipole chicane configuration in which asymmetry and variable $R_{56}$ can be optimized to control CSR-induced emittance growth. This flexibility is achieved by allowing the middle two dipoles to move transversely to the beam and the last dipole to move longitudinally. In this paper we describe the design of the stages used for precise movement of the bunch compressor magnets and associated diagnostics components.
\end{abstract}

\section{INTRODUCTION}

A bunch compressor has been installed [1] in the APS linac in order to achieve a high current of greater than 600 $\mathrm{mA}$ (100-210 MeV), required for the ongoing FEL program. The physics design of the magnetic bunch compressor is based on an asymmetric four-dipole chicane configuration, which is one of the several options proposed by Emma and Bharadwaj [2]. The rms bunch length after compression, $\sigma_{z}$, can be approximated by $\mid 1+$ $k R_{56} \mid \sigma_{z o}$, where $k$ is the RF slope of the precompressor accelerating section, $R_{56}$ is the momentum compaction of the bending section, and $\sigma_{z o}$ is the initial rms bunch length. The bunch is compressed for a positive value of $k$ together with a negative value of $R_{56}$. Performance simulations for the bunch compressor have been carried out in detail by Borland [3] using a tracking code that includes the effects of wakefields and coherent synchrotron radiation (CSR).

\section{BUNCH COMPRESSOR OVERVIEW}

Figure 1 shows schematically important features of the bunch compressor. A precompressor accelerating section (Sector L2) delivers a beam of energy up to $210 \mathrm{MeV}$ to the bunch compressor. The compressor section consists of four chicane dipoles (B1-B4), two "tweaker" quadrupoles, a BPM, a two-blade scraper, and a highprecision $(\sim 10-\mu \mathrm{m})$ flag. The maximum bend angle and corresponding magnetic field of the dipoles are 13.5 degrees and $0.86 T$, respectively. Downstream of the dipole B4, another set of BPM and flag is followed by four matching quadrupoles. A diagnostics section follows, consisting of a three-flag vertical spectrometer magnet and its branch line with two flags, a coherent transition radiation (CTR) diagnostics, and three flags for emittance measurement.

The flexible bunch compressor includes two important features to control beam emittance growth due to CSR: (1) the variable $R_{56}$ can be varied from 0 to $-65 \mathrm{~mm}$ by transverse motion of the B2-B3 dipoles, and (2) the bend angle ratio, i.e. bend angle in dipole pair B1-B2 divided by that in dipole pair B3-B4, can be set to any value between 1 and 1.8. Linear actuators with stepper motors are used to remotely control motions of the B2, B3 and B4 dipoles.

\section{MECHANICAL DESIGN}

\subsection{Design Requirements}

The basic mechanical design requirements established during the conceptual design phase are summarized as follows:

1. Dipoles B2 and B3, each weighing $330 \mathrm{~kg}$, are to move together transversely from 0 to $184 \mathrm{~mm}$ (corresponding $R_{56}$ value of 0 to $-65 \mathrm{~mm}$ ) with a resolution $25 \mu \mathrm{m}$. Diagnostics components between the two dipoles are to maintain their relative positions with respect to the dipoles.

2. Dipole B4, also weighing $330 \mathrm{~kg}$, is to move longitudinally up to $602 \mathrm{~mm}$ (as measured from its initial position for symmetric chicane including $R_{56}$ of $-65 \mathrm{~mm}$ ) with a resolution of $25 \mu \mathrm{m}$.

3. Tweaker quadrupoles $\mathrm{Q} 1$ and $\mathrm{Q} 2$ (Fig. 1) are to remain at fixed distance $(25.6 \mathrm{~cm})$ from the pivot points of dipoles B1 and B4. For all dipole positions, the magnets are to remain centered within $25 \mu \mathrm{m}$ of the lines joining B1-B2 and B3-B4.

4. Flexible chambers [4] inside the dipoles are to bend with the same radii as the electron trajectory to ensure aperture continuity.

\footnotetext{
*Work supported by the U.S. Department of Energy, Office of Basic Energy Sciences under Contract No. W-31-109-ENG-38

†rotela@aps.anl.gov
} 


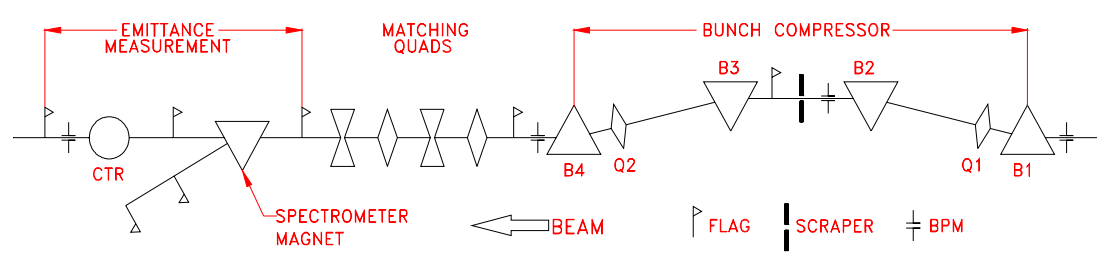

Figure 1: Schematic of the bunch compressor in APS linac.

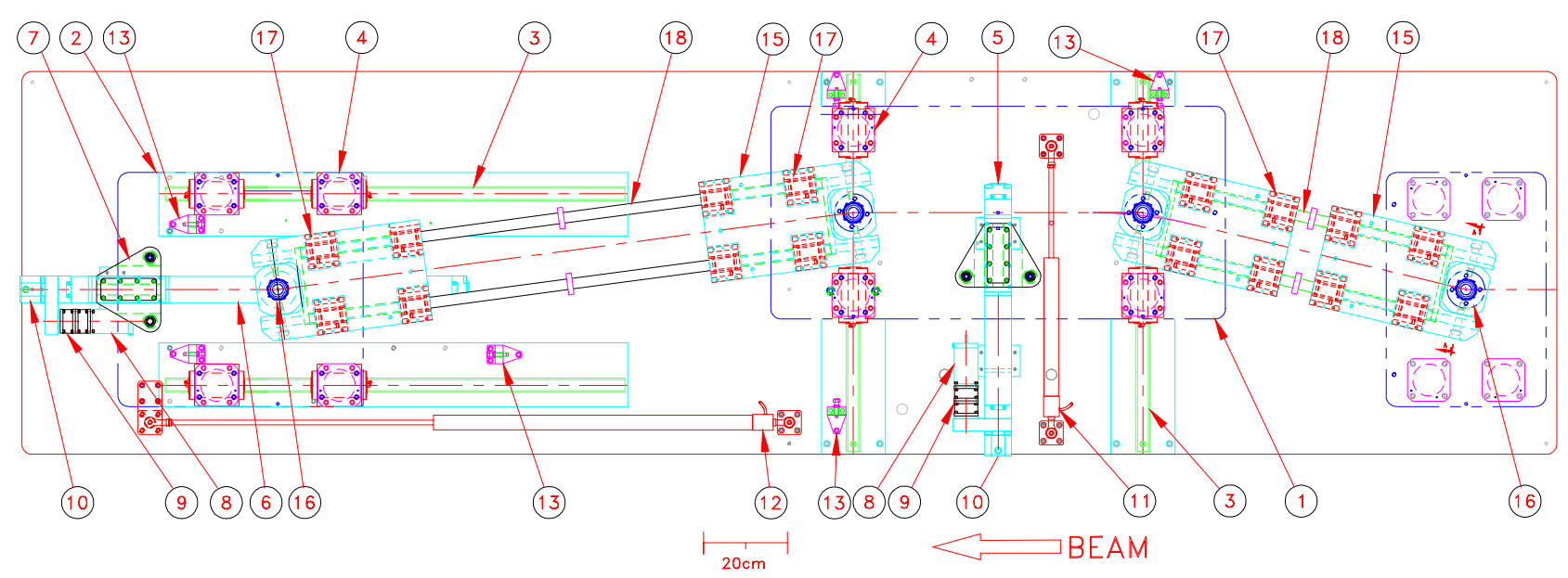

Figure 2: Motion stages of the APS bunch compressor - plan view.

\subsection{Mechanisms for Translation and Rotation}

Figure 2 shows a plan view of the motion mechanisms of the bunch compressor.

The dipole movements are controlled by means of a transverse support table (1) for dipoles B2 and B3, and a longitudinal support table (2) for dipole B4. Both the tables travel on a pair of linear rails (3), each with two linear guideway blocks (4). Two Parker ${ }^{\mathrm{TM}}$ rodless ballscrew actuators (5 and 6), one with a stroke of $200 \mathrm{~mm}$ and another with a stroke of $750 \mathrm{~mm}$, provide the required transverse and longitudinal table motions through connecting links (7). The actuators are driven by stepper motors (8) equipped with helical gear reducers (9) and fail-safe brakes (10). Based on 0.2-inch screw pitch, 200 steps per revolution and 10:1 gear reduction, a linear motion resolution of $1 \mathrm{mil}(25 \mu \mathrm{m})$ is obtained. Hybrid potentiometers (11 and 12) are used for reading positions of the transverse and logitudinal magnet support tables, respectively. Hard stops (13) are provided for safety and to prevent over-travel during assembly and testing phase. The dipole B1, which requires no motion, is mounted on a fixed table.

Two pairs of rotating platforms (15) between B1-B2 and B3-B4 ensure that quadrupoles Q1 and Q2 move as specified in the design requirements. As shown in Fig. 2, each of these platforms is mounted on a pivot assembly (16) and four linear bearing blocks (17). Linear bearing rods (18) connect the platforms in each pair, forcing their centerlines to be co-linear. Q1 and Q2 are mounted on platforms rotating at B1 pivot and B4 pivot, respectively.
The pivot assembly, shown in Fig. 3, uses a pair of 50$\mathrm{mm}$ angular contact ball bearings mounted in tandem on a pivot pin. The ball bearings are assembled inside a bearing housing pressed firmly by a cover plate and a collar nut. The pivot pin is then fastened to the magnet support table and the rotating platform is attached to the bearing housing with shoulder bolts.

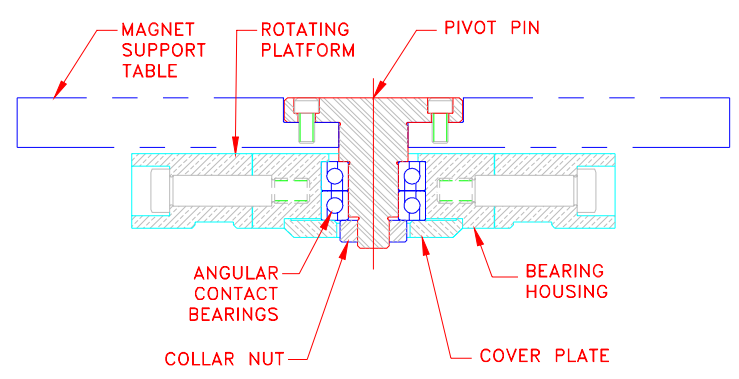

Figure 3: Bunch compressor pivot assembly.

The rotating platforms also ensure that flexible bellows in the dipole magnets conform to the beam trajectory so as not to restrict the chamber aperture. This is accomplished by supporting one end-flange of a flexible bellows on the rotating platform and the second endflange directly on the dipole support table. Similarly, telescopic vacuum chambers [5] between dipoles B1-B2 and $\mathrm{B} 3-\mathrm{B} 4$ are kept in proper alignment by having their supports mounted on the rotating platforms. 


\section{FUTURE DEVELOPMENT}

Initial experimental results with the bunch compressor have been reported by Borland [6]. The mechanisms for moving the magnets have been installed and tested (Fig. 4); however, the magnet motions are not yet possible because the telescopic vacuum chambers require design modifications. These chambers will be installed later in 2001 , allowing optimization of asymmetry and $R_{56}$ to control the emittance growth. At the same time, some components of the beam scraper and the flag will be upgraded to maintain chamber aperture uniformitty.

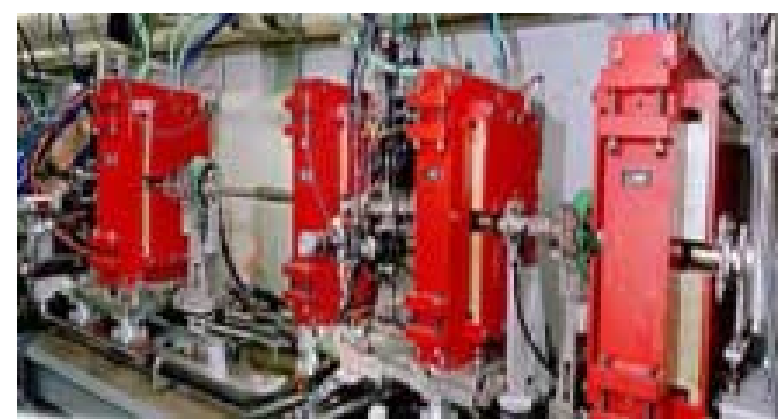

Figure 4: Bunch compressor in APS linac.

Modifications for the beam scraper are depicted in Fig. 5 . The beam aperture in the scraper housing is machined to specified dimensions, $25 \mathrm{~mm}$ horizontal and $23 \mathrm{~mm}$ vertical. The ports for the water-cooled tungsten blades are equipped with rf contact fingers close to the beam aperture. Additionally, a screen is machined in the pumpout port to eliminate abrupt change in the chamber impedance.

A new actuator, shown in Fig. 6, will be used in the highprecision flag. The actuator will be driven by a pair of air

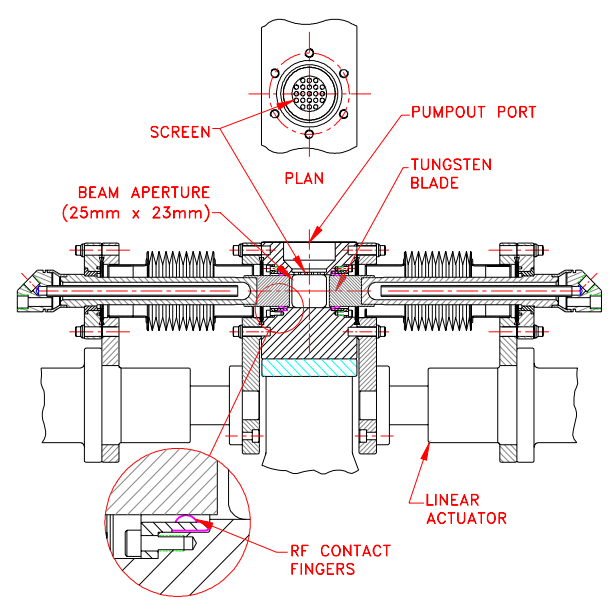

Figure 5: RF contact fingers for the scraper aperture. cylinders allowing four stop positions. A calibration target, a YAG crystal and an optical transition radiation (OTR) are placed in the beam path for the first three positions of the actuator. In the fourth position, a beam aperture $(25 \mathrm{~mm} \times 23 \mathrm{~mm})$ machined in a stainless steel block is centered on the beam axis. As shown in the inset figure, $\mathrm{rf}$ contact fingers are attached to the two sides of the aperture block for $\mathrm{rf}$ continuity.

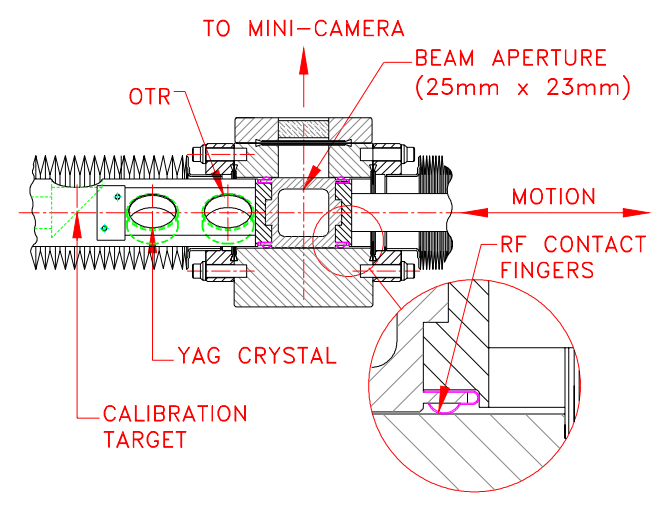

Figure 6: Built-in beam aperture in flag actuator.

\section{ACKNOWLEDGMENTS}

The authors thank N. Arnold, M. Borland, H. Friedsam, S. Milton and B.X. Yang for many helpful discussions. Thanks are also due to $\mathrm{E}$. Theres and $\mathrm{A}$. Kelly for their assistance during the bunch compressor assembly.

\section{REFERENCES}

[1] M. Borland, J. Lewellen, and S. Milton, "A Highly Flexible Bunch Compressor for the APS LEUTL FEL," Proc. LINAC2000 (SLAC) (Aug. 2000).

[2] P. Emma and V. Bharadwaj, "Bunch Compressor Options for the LEUTL Facility at the APS," Technical Note, SLAC (June 8, 1999).

[3] M. Borland, "Design and Performance Simulations of the Bunch Compressor for the APS LEUTL FEL," Proc. LINAC2000 (SLAC) (August 2000).

[4] B.X. Yang, E. Rotela, N. Arnold, J. Stein, W. Berg, and S. Sharma, "Design and Performance of a Compact Imaging System for the APS Linac Bunch Compressor," these proceedings (2001).

[5] D. Walters and L. Morrison, "Flexible and Telescopic Vacuum Chambers for the APS Bunch Compressor," these proceedings (2001).

[6] M. Borland, "APS Bunch Compressor Initial Experimental Results," Presentation at the LCLS TAC Meeting, SLAC (Jan. 12-13, 2001). 